\title{
electronic reprint
}

Acta Crystallographica Section B

Structural

Science

ISSN 0108-7681

Editor: Carolyn P. Brock

Copyright $($ International Union of Crystallography

Author(s) of this paper may load this reprint on their own web site provided that this cover page is retained. Republication of this article or its storage in electronic databases or the like is not permitted without prior permission in writing from the IUCr.

Acta Cryst. (). B, 
Acta Crystallographica Section B

Structural

Science

ISSN 0108-7681

\section{Soraya Maria Belluga \\ Rodriguez, a Lukáš Palatinus, ${ }^{\text {a,b* }}$ Václav Petříček ${ }^{b}$ and Gervais Chapuis $^{\mathrm{a}}$}

aEcole Polytechnique Fédérale de Lausanne, Laboratoire de Cristallographie, BSP, CH-1015 Lausanne, Switzerland, and ${ }^{\mathbf{b}}$ Institute of Physics, Academy of Sciences of the Czech Republic, Na Slovance 2, 18221 Prague, Czech Republic

Correspondence e-mail: palat@fzu.cz

\section{Growth-induced incommensurability observed in the organic co-crystal hexamethylenetetramine resorcinol}

Co-crystals of hexamethylenetetramine and resorcinol were investigated by X-ray diffraction. The structure was refined in the superspace group $X m c m(0 \beta 0) s 0 s, X=\left(\frac{1}{2} \frac{1}{2} 0 \frac{1}{2}\right)$. In the average structure the resorcinol molecules are disordered between two orientations. The main effect of the modulation in the structure is a harmonic modulation of the occupation probabilities of the two orientations of the resorcinol molecule. However, the modulated order is not perfect and the resorcinol molecules remain partially disordered. Below $270 \mathrm{~K}$ the crystal undergoes a phase transition to a commensurately modulated structure with modulation vector $\mathbf{q}=\left(0 \frac{1}{2} \frac{1}{4}\right)$ and superspace group $X 2 / m(0 \beta \gamma) 0 s$. The structure of the low-temperature phase could not be determined owing to the poor quality of the crystals affected by the reconstructive phase transition.

\section{Introduction}

Hexamethylenetetramine, $\left(\mathrm{CH}_{2}\right)_{6} \mathrm{~N}_{4}$ (also called hexamine, urotropine or HMT), is an important agent for the chemical industry in a wide range of applications including rubber production, explosives and disinfectants. Pure hexamine crystallizes in a highly symmetric space group $I \overline{4} 3 m$ (Dickinson \& Raymond, 1923; Gonell \& Mark, 1923). The four N atoms form a tetrahedron, and although four hydrogen bonds could be possible, in organic co-crystals hexamine has the tendency to form only one or two. Only in one case (Jordan \& Mak, 1970) have three hydrogen bonds been observed. Chemists have made systematic studies of self-assembled cocrystals of hexamine with phenols (Jordan \& Mak, 1970; Coupar et al., 1997; Zakaria et al., 2003). A new interest in the chemistry and crystallography of HMT was initiated by the discovery of the incommensurate phase of the co-crystal HMT-suberate (Gaillard et al., 1996). This structure was the first in a series of studies on compounds of the type HMTdicarboxylic acid [HOOC- $\left(\mathrm{CH}_{2}\right)_{n}-\mathrm{COOH}$ ], which exhibit complex behavior including incommensurate phases, twinning, disorder or a combination of these features (Gaillard et al., 1998; Gardon et al., 2003; Bonin et al., 2003; Hostettler et al., 1999).

Motivated by the long-term interest of our laboratory in the crystallography of organic incommensurately modulated crystals in general and in the HMT-containing co-crystals in particular, we have made a survey of potentially interesting crystal structures containing HMT published in the literature. Our attention was drawn to the description of the structure of HMT-resorcinol (Mahmoud \& Wallwork, 1979; CSD refcode 
Table 1

Experimental details.

\begin{tabular}{|c|c|}
\hline \multicolumn{2}{|l|}{ Crystal data } \\
\hline $\begin{array}{l}\text { Crystal ata } \\
\text { Chemical formula }\end{array}$ & $\mathrm{C}_{6} \mathrm{H}_{12} \mathrm{~N}_{4} \cdot \mathrm{C}_{6} \mathrm{H}_{6} \mathrm{O}_{2}$ \\
\hline$M_{r}$ & $\begin{array}{l}\mathrm{C}_{6} \mathrm{H}_{12} \mathrm{~N}_{4} \cdot \mathrm{C}_{6} \mathrm{H}_{6} \mathrm{O}_{2} \\
250.3\end{array}$ \\
\hline Cell setting & Orthorhombic \\
\hline Superspace group & $X m c m(0 \beta 0) s 0 s, X=\left(\frac{1}{2}, \frac{1}{2}, 0, \frac{1}{2}\right)$ \\
\hline q vector & {$[0,0.376(5), 0]$} \\
\hline Temperature (K) & 293 \\
\hline$a, b, c(\AA)$ & $10.3474(14), 7.0763(6), 16.8321(17)$ \\
\hline$V\left(\AA^{3}\right)$ & 1232.5 \\
\hline$Z$ & 4 \\
\hline$D_{x}\left(\mathrm{Mg} \mathrm{m}^{-3}\right)$ & 1.251 \\
\hline Radiation type & Mo $K \alpha$ \\
\hline$\mu\left(\mathrm{mm}^{-1}\right)$ & 0.09 \\
\hline Crystal form, color & Prism, colorless \\
\hline Crystal size (mm) & $0.4 \times 0.3 \times 0.2$ \\
\hline \multicolumn{2}{|l|}{ Data collection } \\
\hline Diffractometer & Stoe IPDS-II \\
\hline Sample-detector distance $(\mathrm{mm})$ & 100 \\
\hline Tube current, voltage $(\mathrm{mA}, \mathrm{kV})$ & 45,55 \\
\hline Absorption coefficient $\left(\mathrm{mm}^{-1}\right)$ & 0.091 \\
\hline Data collection method & $\omega$ scans \\
\hline Scan width $\left(^{\circ}\right)$ & 1.0 \\
\hline Exposure time (s/frame) & 300 \\
\hline Absorption correction & None \\
\hline$T_{\min }$ & - \\
\hline$T_{\max }$ & - \\
\hline $\begin{array}{l}\text { No. of independent reflections (all/ } \\
\text { obs) }\end{array}$ & $2462 / 1097$ \\
\hline $\begin{array}{l}\text { No. of independent main reflections } \\
\text { (all/obs) }\end{array}$ & 919/681 \\
\hline $\begin{array}{l}\text { No. of independent first-order } \\
\text { satellites (all/obs) }\end{array}$ & $1543 / 416$ \\
\hline Criterion for observed reflections & $I>3 \sigma(I)$ \\
\hline Redundancy & 10.9 \\
\hline$R_{\text {int }}(\mathrm{all} / \mathrm{obs})$ & $8.45 / 7.75$ \\
\hline$\theta_{\max }\left({ }^{\circ}\right)$ & 29.4 \\
\hline \multicolumn{2}{|l|}{ Refinement } \\
\hline Refinement on & $F$ \\
\hline \multicolumn{2}{|l|}{$R_{\mathrm{obs}} / w R_{\mathrm{obs}}$} \\
\hline All reflections & $0.0435 / 0.0605$ \\
\hline Main reflections & $0.0388 / 0.0583$ \\
\hline First-order satellites & $0.0674 / 0.0679$ \\
\hline Goodness of fit (obs/all) & $2.14 / 1.66$ \\
\hline No. of reflections & 2462 \\
\hline No. of parameters & 86 \\
\hline $\mathrm{H}$-atom treatment & Constrained to parent site \\
\hline Weighting scheme & $w=1 /\left[\sigma^{2}(F)+0.0004 F^{2}\right]$ \\
\hline$(\Delta / \sigma)_{\max }$ & $<0.0001$ \\
\hline$\Delta \rho_{\max }, \Delta \rho_{\min }\left(\mathrm{e} \AA^{-3}\right)$ & $0.20,-0.13$ \\
\hline Extinction method & $\begin{array}{l}\text { Isotropic type I Gaussian (Becker \& } \\
\text { Coppens, 1974) }\end{array}$ \\
\hline Extinction coefficient & $4.2(3)$ \\
\hline Source of atomic scattering factors & $\begin{array}{l}\text { International Tables for Crystallo- } \\
\text { graphy, Vol. C }\end{array}$ \\
\hline
\end{tabular}

Computer programs used: $X$-AREA (Stoe \& Cie, 2005), SHELXS97 (Sheldrick, 1997), JANA2000 (Petříček et al., 2000), CrystalMaker (CrystalMaker Software, 2006).

RSHMTA). This structure was described in a non-centrosymmetric orthorhombic space group $C 2 m c$ and included disorder of both the HMT and the resorcinol molecules. Moreover, a few weak reflections were reported that could not be indexed by integer indices in the selected lattice. In the present study we present a redetermination of the roomtemperature structure of HMT-resorcinol. It will be shown that the true structure is simultaneously incommensurately modulated and disordered, which is similar to some of the previously studied HMT co-crystals. Recently the structure of HMT-resorcinol was redetermined in the space group $\mathrm{Cm} \mathrm{cm}$ ( $\mathrm{Ng}$ et al., 2002), but this reinvestigation also did not discover the modulated character of the structure.

\section{Experimental}

Equimolar quantities of HMT (318 mg, Fluka, 99.5\%) and resorcinol (250 mg, Riedel-de Haën, >99\%) were separately dissolved in equal amounts of bidistilled water, and later mixed together without stirring. Crystals were grown by slow evaporation at $280 \mathrm{~K}$. Only total volumes of water between 3 and $5 \mathrm{ml}$ yielded crystals. The exact volume of water considerably influences the macroscopic appearance of the crystals. Most of the crystals contain internal channels and inclusions of the solvent. These inclusions may become colored with time. The number and size of the inclusions tend to be smaller when a larger amount of water is used. The whole process of crystal growth had to be performed in a nitrogen atmosphere. If the solution is left in contact with air, a brown coloration appears owing to the oxidation of resorcinol. Crystals grow in the form of plates normal to [100]. Several crystals were carefully cut to obtain monocrystals with approximately equal dimensions, and these crystals were tested for the quality of their diffraction patterns. Most of the crystals diffracted poorly.

A monocrystal with the dimensions $0.2 \times 0.3 \times 0.4 \mathrm{~mm}^{3}$, which produced the sharpest and most intense diffraction spots, was measured at $292 \mathrm{~K}$ on a Stoe IPDS-II image-plate diffractometer using a conventional X-ray tube with Mo $K \alpha$ radiation (see Table 1 for further details).

Measured frames were analyzed with the software $X$-AREA (Stoe \& Cie, 2005). The strongest reflections could be indexed in an orthorhombic cell with lattice parameters $a=$ 10.3474 (14), $b=7.0763$ (6), $c=16.8321$ (17) $\AA$, in accordance with the previously published structure (Mahmoud \& Wallwork, 1979). However, additional reflections were observed at incommensurate positions. These satellite reflections could be indexed by four integers as $\mathbf{h}=h \mathbf{a}^{*}+k \mathbf{b}^{*}+l \mathbf{c}^{*}+m \mathbf{q}$ with the modulation vector $\mathbf{q}=[0,0.376(5), 0]$. Only first-order satellites $(|m|=1)$ had significant intensity.

In addition to the sharp main and satellite reflections, structured diffuse scattering was present in the diffraction patterns of the tested crystals in the form of streaks originating in the satellite reflections and extending approximately along c* $^{*}$ Two streaks combine in many cases to form a characteristic arc (Fig. 1a). No diffuse scattering was observed at the position of the main reflections. The diffuse scattering was relatively weak compared with the intensities of the Bragg reflections and did not prevent a reliable integration of the intensities. The diffuse scattering will be discussed in $\$ 4$.

Another crystal was used to perform short measurements at different temperatures with the primary aim of observing the dependence of the length of the modulation vector on temperature. Eight measurements between 297 and $270 \mathrm{~K}$ were performed on a Kuma four-circle diffractometer with kappa geometry and a Sapphire II CCD detector. The measurements showed that the length of the modulation 


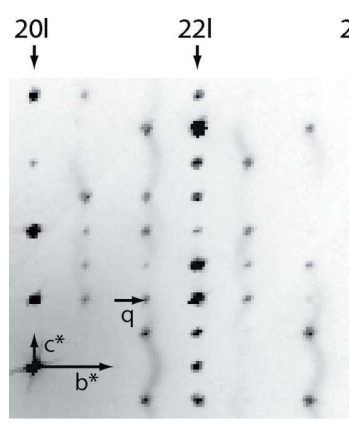

(a)

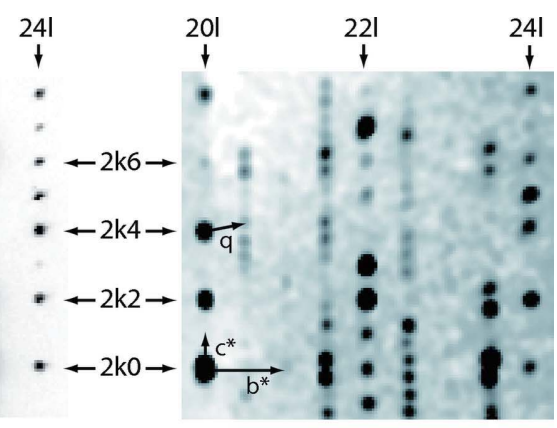

(b)
Figure 1

Part of the $2 \mathrm{kl}$ plane of the diffraction pattern of HMT-resorcinol reconstructed from the area-detector images: $(a)$ at room temperature and $(b)$ at $120 \mathrm{~K}$. For the data collection parameters of $(a)$ see Table 1 ; parameters for $(b)$ : Kuma four-circle diffractometer, Sapphire II CCD detector, Mo $K \alpha$ radiation, sample-detector distance $65.25 \mathrm{~mm}, \omega$ scans, width $0.5^{\circ}$ /frame, exposure time $50 \mathrm{~s} /$ frame.

vector remains constant within one standard deviation and does not show any trend. At 268 (3) K the crystal underwent a phase transition towards a phase with the commensurate modulation vector $\mathbf{q}=\left(0 \frac{1}{2} \frac{1}{4}\right)$. This $\mathbf{q}$ vector remained constant upon further cooling down to $120 \mathrm{~K}$. A preliminary data collection of this low-temperature phase was attempted at $120 \mathrm{~K}$. Unfortunately, the crystal quality decreased severely at the phase transition and the data collection did not produce data of sufficient quality for structure determination of this low-temperature phase. All subsequent attempts to cool a crystal of HMT-resorcinol and to obtain a good data set for the low-temperature phase also failed owing to the severe deterioration of the crystal quality at the phase transition. However, basic information about the space group could be extracted from the measurement and will be discussed in $\$ 4$. It may be possible to overcome the problem with the crystal quality by crystallization at lower temperature directly in the low-temperature phase, but this approach has not been attempted within this project.

\section{Structure solution and refinement}

\subsection{Assignment of the superspace group}

The reflection conditions derived from visual inspection of reconstructed sections of the diffraction pattern are $h \mathrm{klm}$ : $h+k+m=2 n$, corresponding to a non-standard centering $X$ with the centering vector $\left(\frac{1}{2} \frac{1}{2} 0 \frac{1}{2}\right) ; 0 \mathrm{klm}: m=2 n ; h 0 l 0: l=2 n$; $h k 0 m: m=2 n$. This combination of reflection conditions implies a unique superspace group $X m c m(0 \beta 0) s 0 s$. All refinements were performed in this superspace group. The non-standard centering could be transformed into a standard $C$-centering by selecting a $\mathbf{q}$ vector $\mathbf{q}^{\prime}$ with $\beta^{\prime}=1-\beta$. However, this would lead to a $\mathbf{q}$ vector outside the first Brillouin zone. In order to keep the $\mathbf{q}$ vector within the first Brillouin zone we decided to use the non-standard $X$-centering. The space group $X m c m(0 \beta 0) s 0 s$ corresponds to a standard setting Amma(00\%)ss0, No. 63.11 in International Tables for Crystallography (1992, Vol. C, p. 797). Mahmoud \& Wallwork (1979) in their solution of the average structure propose a space group $C 2 \mathrm{~cm}$. However, a closer look at their structure shows that the supposedly missing mirror plane is almost present. The differences in the positions of the atoms related by the mirror planes mimic the effect of strongly anisotropic thermal displacements, which are caused by refining a modulated structure using only main reflections. The extinction condition $0 \mathrm{klm}: m=2 n$ clearly observed in the complete diffraction pattern is proof that the structure contains a mirror plane perpendicular to $\mathbf{a}$.

\subsection{Average structure}

The average structure was solved from main reflections in the space group $\mathrm{Cmcm}$ by direct methods using the program SHELXS97 (Sheldrick, 1997). The initial structure model was further refined with SHELXL97 (Sheldrick, 1997). The refinement converged to residual factors $R(\mathrm{obs})=$ 


\section{research papers}

\section{Table 2}

Modulation parameters of the first harmonic modulation wave of resorcinol and HMT molecules.

$o$ refers to the occupation modulation, $t$ refers to the translational modulation and $r$ refers to the rotational modulation. Subscripts denote the modulation direction, and superscripts $s$ and $c$ refer to the sine and cosine terms, respectively.

\begin{tabular}{lcc}
\hline Parameter & Resorcinol & HMT \\
\hline$o_{s}$ & $0.280(2)$ & - \\
$t_{x}^{s}$ & $-0.00010(15)$ & 0 \\
$t_{y}^{c}$ & $0.0058(2)$ & 0 \\
$t_{z}^{c}$ & $0.00159(9)$ & 0 \\
$r_{x}^{s}$ & $0.00053(11)$ & 0 \\
$r_{y}^{s}$ & 0 & $0.00455(8)$ \\
$r_{y}^{c}$ & $0.00937(11)$ & $-0.00693(8)$ \\
$r_{z}^{c}$ & $0.00098(4)$ & 0 \\
\hline
\end{tabular}

$0.0754, R_{w}(\mathrm{obs})=0.1136$. All atoms were freely refined, except for the $\mathrm{H}$ atoms bonded to $\mathrm{C}$ atoms, which were fixed using the riding model and a distance of $1.0 \AA$ to the parent $\mathrm{C}$ atom. The O1-H10 (hydroxyl group) distance was also restrained to $1.0 \AA$. The average structure obtained by this refinement exhibits a disorder of the resorcinol molecule. The twofold symmetry axis of the resorcinol molecule coincides with the crystallographic twofold axis $2_{x}$ and the center of the aromatic ring lies about $0.3 \AA$ from the mirror plane $m_{x}$. This mirror plane is responsible for the disorder, creating two orientations of the molecule, denoted $A$ and $B$ hereafter (Fig. 2). The two mirror planes of the HMT molecule coincide with the mirror planes $m_{x}$ and $m_{z}$ in the average structure. The average structure is in agreement with previously published results (Mahmoud \& Wallwork, 1979; Tse et al., 1977; Ng et al., 2002).

\subsection{Modulated structure}

The modulated structure was refined using the program JANA2000 (Petrríček et al., 2000). The modulation of individual atoms could not be refined independently, because the limited data set did not allow the refinement of the large number of parameters necessary for such a description. Instead, only the basic positions of the atoms were refined freely, and the modulation of the molecules was refined using the rigid-body approximation. This model significantly reduces the number of refined parameters, because for each molecule only 12 parameters of positional modulation (six for translation and six for rotation) per harmonic wave can be refined. This number is reduced even further if the molecules are located on a special symmetry position, which is the case for both the resorcinol and the HMT molecules. This model is also crystallographically reasonable, since the molecules are rigid and are not expected to deform with the modulation. The number of refined harmonic modulation waves was restricted to one. It is theoretically possible to refine higher harmonic modulation with only the first-order satellites, but in practice this requires either very strong modulation or extremely precise data. Neither of these two requirements was fulfilled in the present case and therefore the refinement with higher harmonics was not attempted.

The presence of the disorder in the average structure suggested an occupational modulation of the resorcinol molecule. Harmonic modulation of the occupation probability $o$ was therefore refined with the restriction $o_{A}+o_{B}=1$. The refinement yielded the following result for the occupational probability of molecule $A$

$$
o_{A}\left(x_{4}\right)=0.5+0.280(2) \sin \left(x_{4}\right)
$$

The maximal and minimal occupations do not reach 1 and 0 , respectively, indicating a substantial degree of disorder even in the modulated structure. It might be tempting to believe that the true form of the occupational modulation is a crenel function. Such modulation would lead to a discontinuous switching between the two orientations of the molecule and the structure would be fully ordered. However, the refinement result with the harmonic occupation already disqualifies this model. This is because a refinement of a crenel function using only one harmonic wave would lead to peak occupancies significantly higher than 1 . Despite this indication, refinement with a crenel function was attempted. It led to significantly worse $R$ values for satellites and confirmed that the harmonic occupation is the most appropriate description of the resorcinol molecule. The occupational modulation is accompanied by a small translational modulation and a rotation of the molecule mainly around b. The modulation of the HMT molecule was described by pure displacement modulation. Owing to the high symmetry of the position of HMT, only the rotation of the molecule around $y$ is possible, all other modulation parameters being zero because of the symmetry restrictions. Introduction of the occupational modulation of the HMT molecule did not lead to an improvement of the $R$ values - rather, it led to an unstable refinement with convergence to false minima. Therefore, this model was excluded.

The structural model just described converged to $R$ values $R_{\text {obs }}$ (main) $=0.0383$ for the main reflections and $R_{\text {obs }}(\mathrm{sat})=$ 0.0681 for the satellites. Further experimental and refinement details are summarized in Table 1.

The modulation parameters of both molecules are summarized in Table 2. The complete set of refined structure parameters has been deposited. ${ }^{\mathbf{1}}$

\section{Discussion}

\subsection{Description of the average structure}

The structure consists of alternating layers of HMT and resorcinol molecules stacked along c. The HMT molecules form a densely packed layer, but the molecules within the layer interact mainly via weak van der Waals interactions. The candidates for hydrogen bonds within the HMT layer are of the type $\mathrm{C}-\mathrm{H} \cdots \mathrm{N}$ and exhibit $\mathrm{H} \cdots \mathrm{N}$ distances longer than

\footnotetext{
${ }^{1}$ Supplementary data for this paper are available from the IUCr electronic archives (Reference: SN5041). Services for accessing these data are described at the back of the journal.
} 
Table 3

Basic characteristics of the hydrogen bonds.

The two numbers in the columns of distances and angles represent the minimum and maximum values along the whole interval of $t$. The distance $D-\mathrm{H}$ was restrained to $1.0 \AA$.

\begin{tabular}{lllll}
\hline $\begin{array}{l}\text { Bonded } \\
\text { molecules }\end{array}$ & $D-\mathrm{H} \cdots A$ & $\mathrm{H} \cdots A(\AA)$ & $\mathrm{D} \cdots A(\AA)$ & $D-\mathrm{H} \cdots A\left({ }^{\circ}\right)$ \\
\hline res-HMT & $\mathrm{O} 1-\mathrm{H} 10 \cdots \mathrm{N} 2^{\mathrm{i}}$ & $1.777-1.810(18)$ & $2.732-2.775(4)$ & $158.5-165.4(12)$ \\
res-HMT & $\mathrm{C} 2-\mathrm{H} 12 \cdots \mathrm{N} 2^{\mathrm{i}}$ & $3.058-3.223(3)$ & $3.694-3.823(3)$ & $119.8-122.7(3)$ \\
res-res & $\mathrm{C} 3-\mathrm{H} 13 \cdots \mathrm{O} 1^{\mathrm{ii}, i i i}$ & $2.699-2.936(9)$ & $3.492-3.672(5)$ & $129.3-139.3(3)$ \\
res-res & $\mathrm{C} 4-\mathrm{H} 14 \cdots \mathrm{O} 1^{\mathrm{iv}, \mathrm{v}}$ & $2.338-2.568(8)$ & $3.035-3.219(6)$ & $120.1-129.3(4)$ \\
HMT-HMT & $\mathrm{C} 6-\mathrm{H} 16 \cdots \mathrm{N} 1^{\mathrm{vi}}$ & $3.197-3.198(15)$ & $4.089-4.090(2)$ & $152.7-152.9(12)$ \\
HMT-HMT & $\mathrm{C} 7-\mathrm{H} 17 \cdots \mathrm{N} 1^{\mathrm{vi}}$ & $3.032-3.198(2)$ & $3.972-4.098(2)$ & $150.4-156.7(1)$ \\
\hline
\end{tabular}

Symmetry codes: (i) $-\frac{1}{2}+x, \frac{1}{2}+y, \frac{1}{2}-z$; (ii) $\frac{1}{2}-x, \frac{1}{2}-y,-z$; (iii) $\frac{1}{2}-x,-\frac{1}{2}+y, z$; (iv) $-\frac{1}{2}+x$, $-\frac{1}{2}+y, z ;(\mathrm{v})-\frac{1}{2}+x, \frac{1}{2}-y,-z ;$ (vi) $\frac{1}{2}-x,-\frac{1}{2}+y, z$.

$3 \AA$ (Table 3). This distance qualifies them as very weak interactions.

The molecules in the resorcinol layer are tilted and packed like tiles on a roof. Each resorcinol position can be occupied by either of two orientations of the molecule. The probability of finding a particular orientation is harmonically modulated with a maximum of $c a$ 0.78. Thus, a substantial degree of disorder is present in the structure, together with the modulation. There are two kinds of hydrogen bonds involving the resorcinol molecules, namely $\mathrm{O}-\mathrm{H} \cdots \mathrm{N}$ hydrogen bonds between the resorcinol and HMT sheets, and weak hydrogen

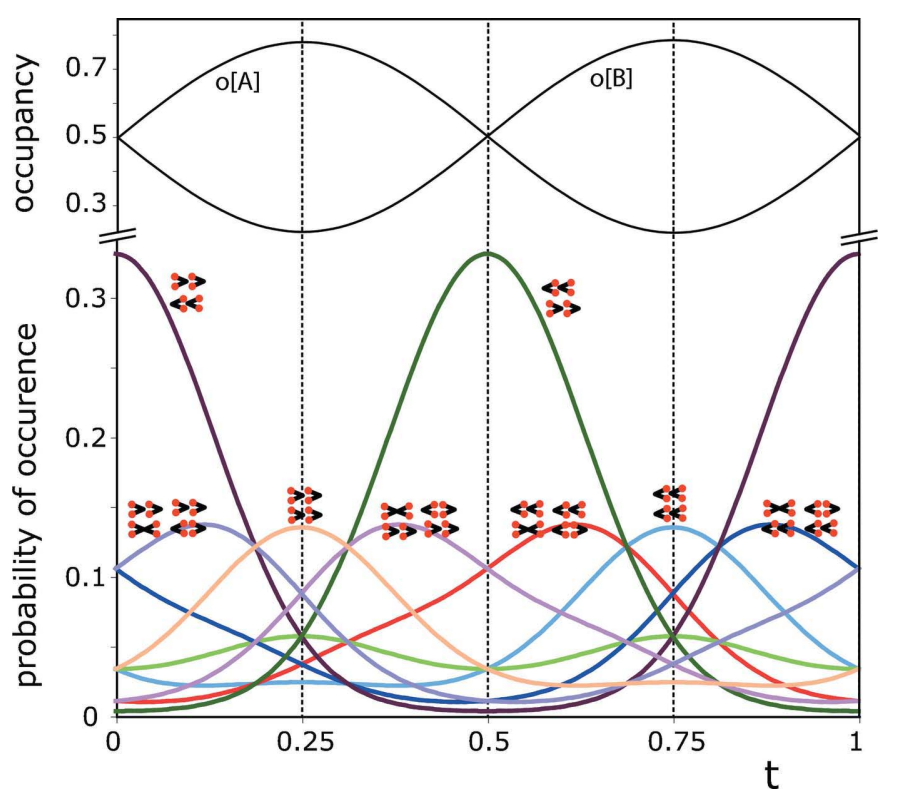

Figure 3

Probabilities of different neighborhoods as a function of $t$. The occupancy of the $A$ and $B$ orientations of the central molecule (o $[A]$ and $\mathrm{o}[B]$, respectively) is shown in the upper part of the figure. The pictograms at each curve schematically represent the orientations of the molecules in the neighborhoods. The orientation of the red dots symbolizes the orientation of the oxygen side of the corresponding resorcinol molecule. See Fig. 4 for an example. The four neighborhoods that are not depicted explicitly belong to the unlabeled curve with low probability. bonds of the type $\mathrm{C}-\mathrm{H} \cdots \mathrm{O}$ linking the resorcinol molecules together within the layers (Table 3). As will be shown in the following paragraphs, the former type plays a key role in the formation of the disorder in the resorcinol sheet, while the latter participates in the formation of the occupational modulation of the resorcinol molecules.

\subsection{The orientational disorder}

There is one relatively strong $\mathrm{O}-\mathrm{H} \cdots \mathrm{N}$ hydrogen bond linking the resorcinol and HMT sheets via atoms $\mathrm{O} 1$ and $\mathrm{N} 2$. This is by far the strongest hydrogen bond in the structure. It can be seen in Table 3 that the bond distances remain almost constant over the whole interval of $t$. Thus, this bond is very stable and is almost unaffected by the modulation. The bonding distances are the same for both orientations of the resorcinol molecule. Thus, the strength of this most important intermolecular interaction is not influenced by the orientation of the resorcinol molecule, and an orientational disorder can occur without much energetic cost.

This interaction is the only strong contact between the resorcinol and HMT sheets. Since its geometry is almost independent of the orientation of the involved resorcinol molecule, it cannot play an important role in the ordering within the resorcinol sheet. Thus, the origin of the incommensurate ordering should be sought in the interactions within the resorcinol layer.

\subsection{Hydrogen bonds in the resorcinol sheets and the modulation}

Each resorcinol molecule has four nearest neighbors within the resorcinol sheet. There are two types of hydrogen bonds between neighboring molecules (Table 3). However, as a result of the orientational disorder of all the molecules and the four nearest neighbors of each molecule there are $2^{4}=16$ possible neighborhoods of the central molecule. The number of actual hydrogen bonds depends on the orientation of the central molecule and also differs among different combinations of the neighbors' orientations. Owing to the disorder, which is present even in the modulated structure, each possible neighborhood of the central molecule has non-zero probability of occurring over the whole $t$ range, but this probability is different for different neighborhoods and also varies with the modulation phase.

For a given central molecule, the probability of finding a particular neighborhood at a particular $t$ can be easily calculated as a product of the occupational probabilities of the four neighboring molecules at that $t$. The result of such a calculation is presented in Fig. 3 together with the occupational probability for the central molecule's orientations. It is obvious from the figure that some neighborhoods have high probabilities close to the highest occupational probability of the $A$ orientation of the central molecule, some have peak probability close to the highest occupancy of the $B$ orientation of the central molecule, and some neighborhoods are the most 


\section{research papers}

probable at places where the two orientations have the same probability of occurring.

In order to draw any useful conclusions from Fig. 3, the intermolecular interactions for the different neighborhoods must be analyzed. The total number of hydrogen bonds linking the central molecule to its neighbors depends both on the orientation of the central molecule and on the particular neighborhood. There are 32 different combinations of the orientation of the central molecule with the neighborhoods. ${ }^{2}$ An example of one neighborhood is shown in Fig. 4. By comparing the number of hydrogen bonds for different configurations with Fig. 3, the following observations can be made:

(i) All neighborhoods giving rise to the same configuration of hydrogen bonds for the $A$ and $B$ orientation of the central molecule give curves that are symmetric with respect to the occupancy of the central molecule. Thus, if the neighborhood is neutral with respect to the number and quality of the hydrogen-bond interactions formed by the two orientations of the central molecule, it is also neutral with respect to the preference to one or another orientation.

(ii) The probability of a particular neighborhood is not correlated with the total number of hydrogen bonds that are formed between the central molecule and the neighboring molecules.

(iii) Given a neighborhood, the orientation of the central molecule is more likely to be the one that can form more hydrogen bonds on its oxygen side (compare Figs. 4 and 3 to see an example).

(iv) The neighborhoods with prevailing $B$ orientation are more likely to have an $A$ orientation in the center and vice versa.

The above observations can be summarized in a series of conclusions:

The lack of correlations between the probability of a neighborhood and the total number of hydrogen bonds formed indicate that the ordering is not a result of an equilibrium in the crystal structure but rather a result of the processes during the crystal growth. This indication is strengthened by the observation that for each neighborhood such an orientation is preferred that gives rise to a larger number of hydrogen bonds on the oxygen side of the molecule. We must also consider that the shape of the molecule and its surrounding makes it virtually impossible to change orientation from $A$ to $B$ and back, once its position has been fixed in the crystal. Thus, a scenario can be proposed that during the growth of the crystal the molecules tend to interact with the neighborhood with its chemically active oxygen side. As a result, the molecules will be preferably oriented so as to build as many hydrogen bonds on the oxygen side as possible. Once bonded to the neighboring resorcinol molecules and fixed by the strong hydrogen bonds to the HMT layer, the molecule cannot change its orientation even if the change would lead to a more stable configuration. Although the

\footnotetext{
2 A table with the number of hydrogen bonds for each of the 32 possibilities has been deposited as supplementary material.
}

hydrogen bonds linking the resorcinol molecules in the structure are quite weak (Table 3 ), they could be stronger at the time of the growth and the distances and angles could adjust to their final values as a result of an equilibrium geometry of the final structure, determined to a large extent by the packing of the HMT sheet.

The above scenario has one important consequence, namely that the modulation wave (the length of the modulation vector) should be frozen in the crystal and independent of temperature. The temperature-dependent measurements fully confirm this prediction, since the length of the modulation vector remains trendless and constant within one standard deviation over the investigated temperature range between 265 and $298 \mathrm{~K}(\$ 2)$.

The fact that $B$-rich neighborhoods will in general lead to a more probable occurrence of the $A$ orientation in their vicinity introduces an instability in the system which alone is likely to lead to an occupational ordering within the resorcinol sheet. However, this cannot explain the incommensurate order, because it is possible to build different ordering schemes that comply with the above observations and that are not necessarily incommensurate. Thus, additional long-range coulombic interactions between the resorcinol molecules have to be assumed. The second-nearest neighbors of a central molecule are located at $\pm \mathbf{b}$ and with an average $\mathrm{O} \cdots \mathrm{O}$ distance of only about $4 \AA$ are good candidates for such an interaction. The direction of this contact can also explain why the modulation vector is oriented along $\mathbf{b}$.

\subsection{Diffuse scattering and the low-temperature phase}

The experimental difficulties with the low-temperature (LT) phase (\$2) prevented its structure determination. However,

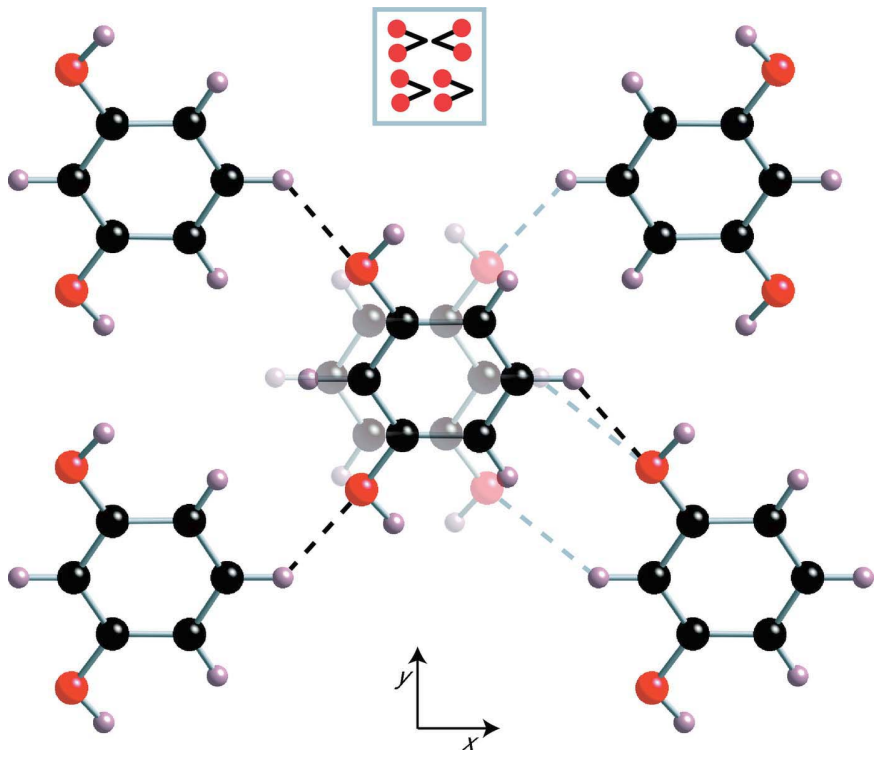

Figure 4

Example of the neighborhood of one resorcinol molecule. The hydrogen bonds formed by the central molecule are shown by dashed lines. The two overlapping molecules in the center show the two possible orientations ( $A$ lighter, $B$ darker). The framed pictogram at the top shows how this neighborhood is represented in Fig. 3. 


\section{research papers}

the measured data were good enough to extract information about the symmetry of the LT phase.

A close inspection of reciprocal-space sections reconstructed from the CCD images showed that the position and overall intensity distribution of the main reflections remained essentially the same, but the incommensurate modulation vector $(0 \beta 0)$ changed to a commensurate vector $\mathbf{q}=\left(0 \frac{1}{2} \frac{1}{4}\right)$ (Fig. 1). The symmetry of the diffraction pattern remains orthorhombic and could be indexed by two modulation vectors $\mathbf{q}_{1}=\left(0 \frac{1}{2} \frac{1}{4}\right)$ and $\mathbf{q}_{2}\left(0 \frac{1}{2}-\frac{1}{4}\right)$, leading to a $(3+2)$ dimensional superspace group. However, no mixed satellites were observed at positions $\mathbf{q}_{1}+\mathbf{q}_{2}$ and $\mathbf{q}_{1}-\mathbf{q}_{2}$, although the first-order satellites are quite strong and the second-order satellites are clearly visible. This strongly suggests that the true symmetry of the low-temperature phase is monoclinic with a single modulation vector $\mathbf{q}=\left(0 \frac{1}{2} \frac{1}{4}\right)$, and the crystal is twinned with the twinning plane (010) or (001).

With this assumption, the following reflection conditions can be deduced from the diffraction pattern: hklm: $h+k=2 n$, which is equivalent to $h+k+m=2 n$ due to the commensurability of the modulation vector, and $0 \mathrm{klm}$ : $m=2 n$. These conditions correspond to the first and second reflection conditions in the incommensurate phase, but the third and fourth conditions from the incommensurate phase are no longer fulfilled, indicating that the symmetry planes perpendicular to $\mathbf{b}$ and $\mathbf{c}$ are no longer present. The superspace group corresponding to the observed conditions is $X 2 / m(0 \beta \gamma) 0 s$ with a as the monoclinic axis. This is a subgroup of the superspace group of the incommensurate structure, but the large change of the modulation vector requires a significant reordering in the structure. The consequence of this reordering is the observed loss of crystallinity at the phase transition and the difficulties in obtaining a good single crystal of the LT phase.

The phase transition occurs at a temperature quite close to room temperature (RT), and traces of the LT phase are visible in the RT phase in the form of diffuse scattering. A comparison of the diffraction patterns of the RT and LT phases (Fig. 1) shows that the diffuse streaks start from the position of the incommensurate satellites and extend towards the positions of the first-order satellites of the LT phase. Typically, the more intense the diffuse streak, the more intense will be the satellite reflection of the LT phase associated with that streak. All these observations lead to the conclusion that a short-range order exists already at room temperature, which is a precursor of the long-range order in the LT phase. The form of the streaks of the diffuse scattering suggests the presence of a stacking disorder. The absence of diffuse streaks at the positions of the main reflections shows that there are no stacking faults in the average structure, but only in the relative phase of the modulation of successive layers.

The phase transition does not have any strong effect on the main reflections. Thus, the basic structure probably remains unchanged and it is the modulation, i.e. mainly the ordering of the orientations of the resorcinol molecules, that is changing. The length of the wavevector projected on to the plane of the resorcinol layers changes at the phase transition, and there- fore the transition cannot be explained only by relative translations of the whole layers but must also include reordering of the molecules within the resorcinol sheets. We have argued above that it is almost impossible for the resorcinol molecules to flip from one orientation to another. The reordering of the orientations at the phase transition must thus proceed with a different mechanism. The sheets of resorcinol molecules are separated by layers of HMT molecules preventing any communication between the resorcinol sheets. The most likely mechanism of the phase transition is the diffusion of the resorcinol molecules within the resorcinol sheets. The diffusion can be initiated at structural defects and can propagate along the resorcinol sheets in the form of a 'hopping' of individual molecules between positions without changing their orientation. This mechanism requires quite severe changes in the structure, which can explain the loss of crystallinity at the phase transition, but at the same time requires much smaller local distortions than would be necessary for flipping of individual resorcinol molecules from one orientation to another.

\section{Conclusions}

The structure of the co-crystal of hexamethylenetetramine and resorcinol is incommensurately modulated at room temperature. The structure has been described in superspace in the $(3+1)$-dimensional superspace group $X m c m(0 \beta 0) s 0 s$. The resorcinol molecule is disordered between two symmetryrelated orientations and the probability of finding the molecule in one of the two orientations varies with the phase of the modulation. This modulation is harmonic rather than steplike. The strongest hydrogen bond connects the resorcinol molecules to the molecules of HMT, and its geometry is independent of the orientation of the resorcinol molecule, thus allowing the disorder to develop. The weaker hydrogen bonds linking the resorcinol sheets appear to be responsible for the ordering within the resorcinol sheet. The analysis of the probability of different neighborhoods of the resorcinol molecules shows that the ordering is not a result of an equilibrium in the crystal structure, but rather occurs during the crystal growth. Under $270 \mathrm{~K}$ the structure undergoes a phase transition towards a monoclinic twinned structure with probably the same basic structure but with different ordering of the resorcinol molecules characterized by the commensurate modulation vector $\mathbf{q}=\left(0 \frac{1}{2} \frac{1}{4}\right)$. The phase transition is assessed as reconstructive and it is argued that the transition proceeds by the diffusion of the resorcinol molecules along the resorcinol sheets. Traces of the low-temperature structure are observable at room temperature in the form of a structured diffuse scattering. The full structure determination of the LT structure could not be performed owing to the deterioration of the crystal at the phase transition temperature.

We are grateful to Michal Dušek for his assistance during the initial stages of this work and to Kurt Schenk for discussions and reviewing the manuscript. The support of this study 


\section{research papers}

by the Swiss National Science Foundation, grant No. 20105325/1, and by the Grant Agency of the Czech Republic, grant No. 202/06/0757, is gratefully acknowledged.

\section{References}

Becker, P. J. \& Coppens, P. (1974). Acta Cryst. A30, 129-153.

Bonin, M., Welberry, T. R., Hostettler, M., Gardon, M., Birkedal, H., Chapuis, G., Möckli, P., Ogle, C. A. \& Schenk, K. J. (2003). Acta Cryst. B59, 72-86.

Coupar, P. I., Glidewell, C. \& Ferguson, G. (1997). Acta Cryst. B53, 521-533.

CrystalMaker Software (2006). CrystalMaker. CrystalMaker Software, Bicester, Oxfordshire, UK.

Dickinson, R. G. \& Raymond, A. L. (1923). J. Am. Chem. Soc. 45, $22-$ 29.

Gaillard, V. B., Chapuis, G., Dušek, M. \& Petříček, V. (1998). Acta Cryst. A54, 31-43.

Gaillard, V. B., Paciorek, W., Schenk, K. \& Chapuis, G. (1996). Acta Cryst. B52, 1036-1047.
Gardon, M., Pinheiro, C. B. \& Chapuis, G. (2003). Acta Cryst. B59, 527-536.

Gonell, H. W. \& Mark, H. (1923). Z. Phys. Chem. 107, 181-218.

Hostettler, M., Birkedal, H., Gardon, M., Chapuis, G., Schwarzenbach, D. \& Bonin, M. (1999). Acta Cryst. B55, 448-458.

Jordan, T. H. \& Mak, T. W. (1970). J. Chem. Phys. 52, 3790-3794.

Mahmoud, M. M. \& Wallwork, S. C. (1979). Acta Cryst. B35, 23702374.

Ng, S. W., Naumov, P., Ibrahim, A. R., Fun, H.-K., Chantrapromma, S., Wojciechowski, G., Brzezinski, B. \& Hanna, J. V. (2002). J. Mol. Struct. 609, 89-95.

Petříček, V., Dušek, M. \& Palatinus, L. (2000). JANA2000. Institute of Physics, Prague, Czech Republic.

Sheldrick, G. M. (1997). SHELXS97 and SHELXL97. University of Göttingen, Germany.

Stoe \& Cie (2005). $X$-AREA. Stoe \& Cie, Darmstadt, Germany. Tse, C.-S., Wong, Y.-S. \& Mak, T. C. W. (1977). J. Appl. Cryst. 10, 6869.

Zakaria, C. M., Ferguson, G., Lough, A. J. \& Glidewell, C. (2003). Acta Cryst. C59, m271-m274. 